\title{
Fano resonant plasmonic systems: Functioning principles and applications
}

\author{
B. Gallinet* , A. Lovera*, T. Siegfried ${ }^{\dagger}$, H. Sigg ${ }^{\dagger}$ and O.J.F. Martin* \\ ${ }^{*}$ Nanophotonics and Metrology Laboratory, Swiss Federal Institute of Technology Lausanne (EPFL), \\ CH-1015 Lausanne, Switzerland \\ ${ }^{\dagger}$ Laboratory for Micro- and Nanotechnology, Paul Scherrer Institut, CH-5232 Villigen-PSI, Switzerland
}

\begin{abstract}
Using a generalization of Fano formula that includes the intrinsic losses associated with metals, we retrieve the underlying modal structure that produces Fano resonances from the interference between a bright and a dark mode. This knowledge is used to determine the most efficient plasmonic system for a specific application by tuning its mode of operation from weak coupling to best energy storage or strong coupling. This approach is illustrated with examples from sensing.
\end{abstract}

Keywords: Plasmons, Fano resonances, Sensing, Nanofabrication PACS: 73.20.Mf, 73.21.-b, 78.67.Bf, 41.20.-q, 02.30.Rz

Over the last few years, Fano resonances have become the subject of vivid interest in the plasmonics community [1]. Most harmonic systems exhibit the Lorentzian lineshape illustrated in the left of Fig. 1(a). In the original work of Ugo Fano in atom spectroscopy, the characteristic asymmetric lineshape that bears his name occurs from the interference between a discrete state with a continuum. In plasmonics, Fano resonances arise from the interaction between a localized (dark) mode and a radiative (bright) mode. While the bright mode can be excited from the far-field under planewave illumination, the dark mode cannot. However, it can be excited by the near-field associated with the bright mode. Hence, the illumination field first excites the bright mode, which then excites the dark mode. In turn, the field of this dark mode interferes with that of the bright mode. Depending on the relative phase between these two pathways, the response of the bright mode is either strengthen or attenuated. Since this relative phase depends on the excitation frequency, the system exhibits an asymmetric lineshape, as illustrated in the right part of Fig. 1(a).

The response of the total system is the product of the bright mode response with the Fano lineshape:

$$
\sigma(\omega)=\frac{\Gamma_{b}^{2}}{\left(\omega^{2}-\omega_{b}^{2}\right)+\Gamma_{b}^{2}} F(\omega),
$$

where $F(\omega)$ represents the Fano lineshape. In the original work of Fano, this function reads

$$
F(\omega)=\frac{(\kappa+q)^{2}}{\kappa^{2}+1}
$$

where

$$
\kappa=\frac{\omega^{2}-\omega_{d}^{2}-\omega_{d} \Delta}{\Gamma}
$$

(a)

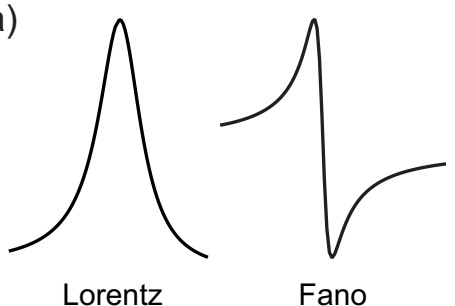

(b)

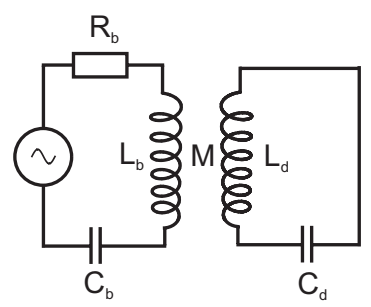

(c)

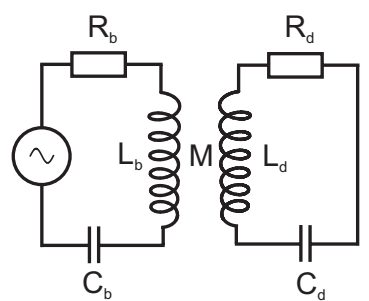

FIGURE 1. (a) Lorentzian and Fano lineshapes. (b) Original circuit equivalent for Fano resonances and (c) equivalent circuit for a plasmonic system.

$$
q=\frac{\omega_{d}^{2}-\omega_{b}^{2}}{\gamma_{b} \omega_{d}}
$$




$$
\begin{aligned}
\Delta & =\frac{\omega_{d}^{2}-\omega_{b}^{2}}{\omega_{d}} \\
\Gamma & =\gamma_{b} \omega_{d} .
\end{aligned}
$$

The frequencies of the bright and dark modes are $\omega_{b}$ and $\omega_{d} ; \gamma_{b}$ represents the losses associated with the bright mode and is represented in Fig. 1(b) by $R_{b}$.

The underlying physics can be easily understood by studying a model of two coupled mechanical oscillators or two coupled electric resonant circuits, as illustrated in Fig. 1(b). The left mesh in this circuit corresponds to the bright mode and is driven by the current source. The resistance $R_{b}$ accounts for radiative losses. The right mesh in the circuit corresponds to the dark mode. In the original work of Fano, described by Eq. (2), no losses were included in this dark mode. This absence of losses represents the main limitation for the application of Fano formula to plasmonic systems, since they exhibit significant ohmic losses, which lead to thermal dissipation of the incident energy.

Figure 1(c) provides a better description for a plasmonic Fano resonant system: an additional resistance $R_{d}$ has now been incorporated in the right mesh of the circuit describing the dark mode. This resistance accounts for ohmic losses in the plasmonic system. Fano formula (2) does not apply in this case and we recently developed a generalized Fano formula for this case [2],

$$
F(\omega)=\frac{(\kappa+q)^{2}+b}{\kappa^{2}+1},
$$

where the new parameter $b$ - the so-called modulation damping parameter - describes the damping of the modulation amplitude of the Fano resonance (when $b$ is large, the Fano dip is very modest). Note that the generalized Fano formula (7) can be derived using different approaches, including the quantum mechanics Feshbach formalism of project operators [3, 2], but also the classical model of two coupled oscillators [4].

In the first experiment dealing with Fano resonant plasmonic nanostructures, experimental or numerical data were fitted with a series of Lorentzian curves including damping terms [5]. This procedure is perfectly appropriate since the underlying system is composed of a series of harmonic oscillators. However, this procedure does not provide any physical insights into the modal structure leading to the Fano resonance. On the other hand, using the generalized Eq. (7) it is possible to retrieve the different parameters associated with the bright and dark plasmonic modes [6]. This procedure is illustrated in Fig. 2, where the response of a dolmen structure composed by two parallel gold nanowires supporting a quadrupole (dark) mode with a third wire supporting a dipole (bright) mode [7]. The structure is made from a Drude metal with plasma frequency $\omega_{p}=1.37 \cdot 10^{16} \mathrm{~s}^{-1}$ and damping $\gamma=0.009 \omega_{p}$. The length of the different
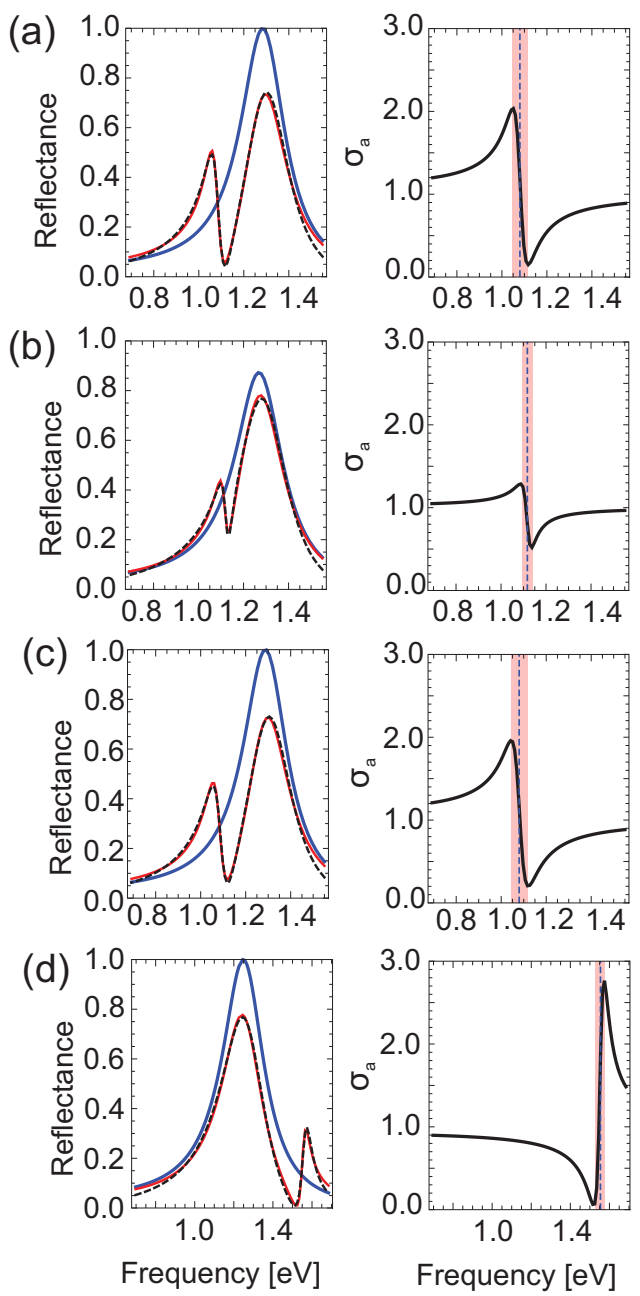

FIGURE 2. Retrieving the modal structure associated with Fano resonances in dolmen structures with varying geometry. For each geometry, the left panel shows the reflectance and the retrieved bright mode, while the right panel shows the retrieved dark mode.

bars is $300 \mathrm{~nm}$ and there is a $30 \mathrm{~nm}$ gap between two parallel wires and the third one. To retrieve the underlying modal structure, we first compute the reflectance from a 2D array composed of such dolmen structures using a surface integral equation with periodic boundary conditions [8]. A similar procedure would be used if the reflectance was measured experimentally, instead of being computed. By fitting Eq. (7) to the reflectance data, it is possible to determine all the parameters that characterize the bright and dark modes producing the Fano resonance. For each case studied in Fig. 2 we show in the left panel the reflectance and the profile of the bright mode, while we show in the right panel the dark mode. Figure 2(a) is the reference geometry. In Fig. 2(b) we de- 

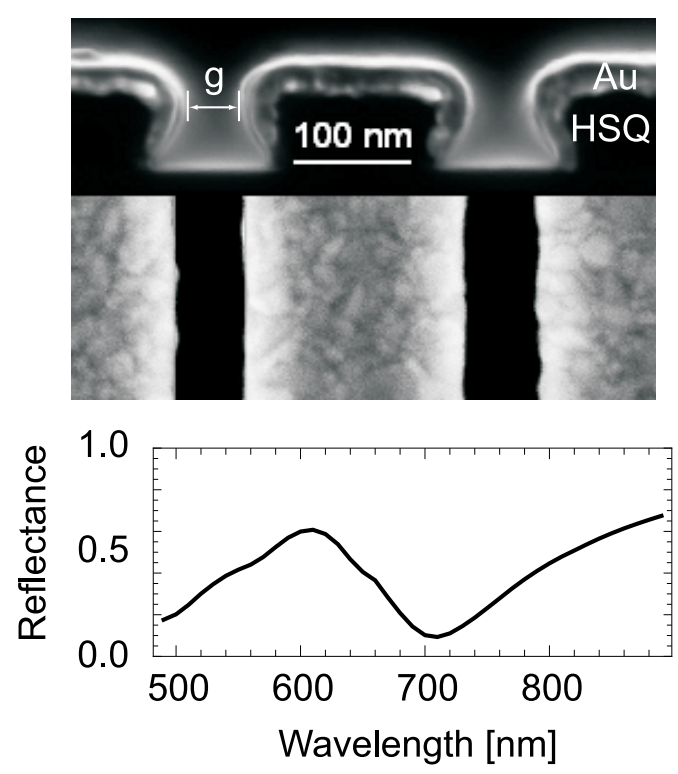

FIGURE 3. Plasmonic Fano resonant system composed of a sub-wavelength grating and the corresponding Fano resonance in the reflectance.

crease the coupling by moving the third nanowire away from the quadrupole; this decreases the modulation of the Fano resonance and the dark mode becomes more narrow. In Fig. 2(c) we increase the losses in the dark mode by increasing the damping in the Drude model; this also reduces the modulation of the Fano resonance and increases the width of the dark mode. Finally, in Fig. 2(d) we shorten the two beams that support the quadrupole mode, which now occurs at a higher energy, leading to a reversed pattern for the Fano resonance, compare with Fig. 2(a).

The previous results illustrate the possibility to gain insights into the mechanisms leading to Fano resonances in plasmonic nanostructures, in particular to understand the link between the Fano lineshape and the properties of the underlying bright and dark modes. This knowledge can also be used to design custom plasmonic nanostructures for specific applications. In this last part of the presentation, we will show how to engineering the bright and dark modes to enhance specific features, and enable the design of Fano resonant structures that operate in different modes, viz. weak coupling, best energy storage, or strong coupling.

Figure 3 shows an array of gold nano-crescents realized by interference lithography [9] and the corresponding reflectance which exhibits a Fano resonance around $700 \mathrm{~nm}$. By using the same procedure as in Fig. 2, it is possible to retrieve the modal structure associated with this system. Furthermore, by modifying the geometrical parameters, it is possible to engineer the Fano resonance so that different coupling regimes can be explored. In particular, we will show that conditions for maximum Raman enhancement for molecules located on the structure can be achieved.

In conclusion, engineering Fano resonances in plasmonic systems for specific applications requires a deep knowledge of the underlying modal structure. We have shown that an appropriate approach for retrieving this modal structure from experimental or numerical data relies on the utilization of the generalized Fano formula, which accounts for ohmic losses in plasmonic metals. This way, it is possible to describe the bright and dark modes that produce the Fano resonance and to engineer them for specific aplications.

Funding from CCMX-Fanosense, the Swiss National Science Foundation and the European CommunityŠs Seventh Framework Program (FP7-ICT-2009-4, Grant agreement 248835) is gratefully acknowledged. Part of this work was performed at the Swiss Light Source (SLS), PSI, Switzerland.

\section{REFERENCES}

1. B. Luk'yanchuk, N. I. Zheludev, S. A. Maier, N. J. Halas, P. Nordlander, H. Giessen, and C. T. Chong, "The Fano resonance in plasmonic nanostructures and metamaterials," Nat. Mater. 9, 707-715 (2010).

2. B. Gallinet and O. J. F. Martin, "Ab initio theory of Fano resonances in plasmonic nanostructures and metamaterials," Phys. Rev. B 83, 235427 (2011).

3. D. J. Bergman and M. I. Stockman, "Surface Plasmon Amplification by Stimulated Emission of Radiation: Quantum Generation of Coherent Surface Plasmons in Nanosystems," Phys. Rev. Lett. 90, 027402 (2003).

4. G. Gallinet, "Fano resonances in plasmonic nanostructures: Fundamentals, numerical modeling and applications," $\mathrm{PhD}$ Thesis 5299, EPFL (2012).

5. A. Christ, Y. Ekinci, H. H. Solak, N. A. Gippius, S. G. Tikhodeev, and O. J. F. Martin, "Controlling the Fano Interference in a Plasmonic Lattice," Phys. Rev. B 76, 201405 (2007).

6. B. Gallinet and O. J. F. Martin, "Influence of electromagnetic interactions on the line shape of plasmonic Fano resonances," ACS Nano 5, 8999-9008 (2011).

7. N. Liu, H. Guo, L. Fu, S. Kaiser, H. Schweizer, and H. Giessen, "Three-dimensional photonic metamaterials at optical frequencies," Nat. Mater. 7, 31-37 (2008).

8. B. Gallinet, A. M. Kern, and O. J. F. Martin, "Accurate and versatile modeling of electromagnetic scattering on periodic nanostructures with a surface integral approach," J. Opt. Soc. Am. A 27, 2261-2271 (2010).

9. T. Siegfried, Y. Ekinci, H. H. Solak, O. J. F. Martin, and H. Sigg, "Fabrication of sub-10 nm gap arrays over large areas for plasmonic sensors," Appl. Phys. Lett. 99, 263302 (2011). 